\title{
Development and evaluation of a statewide autopsy education workshop
}

\author{
Cathleen M Connell, PhD • Sara B. Holmes, MPH • Holly Avey, MPH
}

\section{Abstract}

The role of autopsy is critical in confirming diagnoses and advancing research in Alzheimer's disease. In collaboration with the Alzheimer's Association chapters in Michigan, the staff of the Education Core of the Michigan Alzheimer's Disease Research Center developed, implemented, and evaluated an educational intervention based on a train-the-trainer model to disseminate information about autopsy to chapter staff and volunteers. Participants at a one-day Autopsy Education Workshop (AEW) developed skills to make presentations about autopsy in their local communities and assist family caregivers with decision-making. Participants reported significant gains in knowledge about autopsy and an increased level of confidence in discussing autopsy with family members. The AEW curriculum and evaluation design will be described.

\section{Introduction}

Autopsy plays an important role in understanding Alzheimer's disease (AD). Results of brain autopsies make it possible to confirm the clinical diagnosis of $\mathrm{AD},{ }^{1}$ assess the efficacy of medications and treatments, ${ }^{2}$ provide data for registries of rates of prevalence and mortality, ${ }^{3}$ and enable researchers to better understand the genetic aspects of the disease. ${ }^{4}$ Families of people with AD report several benefits of having an autopsy performed, including the advancement of research and medical knowledge ${ }^{5,6,7}$ and reassurance that appropriate care was given while the patient was alive. ${ }^{5,6}$ Additionally, autopsy results can be used to obtain a definitive diagnosis that will inform other family members about their personal risk of developing $\mathrm{AD}$ in the future. ${ }^{7,8}$

Cathleen M Connell, PhD, Director, Education and Information Transfer Core, Michigan Alzheimer's Disease Research Center, University of Michigan, Ann Arbor, Michigan.

Sara B. Holmes, MPH, Senior Health Educator, Michigan Alzheimer's Disease Research Center, University of Michigan, AnnArbor, Michigan. Holly Avey, MPH, Health Educator, Michigan Alzheimer's Disease Research Center, University of Michigan, Ann Arbor, Michigan.
Despite these benefits, family members report numerous barriers to having an autopsy performed. Results of mailed questionnaires and in-depth interviews indicate that cost, a lack of family agreement about whether the procedure should be performed, concern about disfigurement of the body, lack of information from physicians about the procedure, and perceived delay of viewing, funeral, and burial arrangements serve as significant barriers to autopsy for many family members. ${ }^{5,6,9}$ These tangible and attitudinal barriers as well as misconceptions about autopsy need to be addressed in efforts to disseminate information about autopsy and develop appropriate educational interventions. As reported in a recent study, ${ }^{5}$ the most critical educational needs related to autopsy include facilitating family decision-making, increasing knowledge of autopsy procedures, and providing assistance with accessing local resources and planning an autopsy.

The present study describes the development, implementation, and evaluation of an Autopsy Education Workshop (AEW)conducted by the Education and Information Transfer Core (Education Core) of the Michigan Alzheimer's Disease Research Center (MADRC). This outreach activity was conducted in collaboration with the Executive Directors of the 11 Alzheimer's Association chapters in Michigan and coordinators of the Michigan Dementia Postmortem Network (subsequently referred to as the Network). This statewide network, established in 1987, coordinates the efforts of medical professionals and volunteers at selected sites throughout Michigan to assist families in the process of planning and obtaining an autopsy. ${ }^{10}$

As part of the Network, referral liaisons, usually a social worker, nurse or Alzheimer's Association chapter volunteer, discuss autopsy issues with the family and assist them in completing the appropriate paperwork to register the patient with the Network. Autopsy liaisons, usually a pathology assistant, coordinate the patient registry forms and coordinate the brain removal with the 
pathologist at a local hospital. Other key members of the Network include pathologists and neuropathologists. Pathologists review the registry forms and remove the brain tissue. Neuropathologists conduct an examination of the brain tissue, make a diagnosis, and send an autopsy report to the patient's physician and family.

The three major goals of the AEW were:

- To provide training to at least one staff member or volunteer from each of the local chapters of the Alzheimer's Association in Michigan to serve as local autopsy educators;

- To provide training to key participants in the Network, including referral liaisons and autopsy liaisons.

- To develop an autopsy manual to accompany the Workshop.

\section{Program development}

In April 1993, the staff of the Education Core was invited to present the results of a study conducted to examine attitudes toward autopsy ${ }^{5}$ to the Chapter Directors of the Michigan Alzheimer's Association. Several key themes were identified as part of this study, including misconceptions about autopsy procedures, difficulties with family decision-making, concern about the cost of the procedure, and religious objections to autopsy. Because these concerns reflected the challenges faced by the local chapters of the Alzheimer's Association in disseminating information about autopsy, the Chapter Directors expressed interest in forming a planning committee to develop an educational intervention to address the needs of families interested in learning more about autopsy. This planning committee included two master's level health educators employed by the Education Core and seven of the Chapter Directors of the Alzheimer's Association in Michigan, many of whom also serve as Network volunteers. Representatives of each of the eleven chapters of the Alzheimer's Association in Michigan were invited to serve on the planning committee. Although all chapters expressed interest in an autopsy education intervention, four chapters in rural areas of the state were unable to participate due to personnel shortages and the cost of travel to attend meetings.

The first planning committee meeting was held in July 1993, in Ann Arbor, Michigan. Committee members generated a list of key topics to be addressed in an educational intervention and decided to coordinate their efforts with the services provided by the Network. A train-the-trainer format was selected as appropriate for a one-day workshop to be held in the Spring of 1994.
During a second planning committee meeting in September 1993, the topics to be covered during the workshop and selection of presenters was finalized. The workshop curriculum was developed by the health educators of the Education Core, in close collaboration with a neuropathologist who has been involved in the Network since its inception. Information gathered during telephone calls and follow-up mailings was used to finalize the workshop brochure, role-play scenarios, handouts describing local resources, and the selection of the date and place for the workshop. To strengthen the coordination of autopsy services in local communities, a decision was made to direct the training to two target audiences-volunteers of the Alzheimer's Association, (who would then serve as autopsy educators), and Network volunteers (who would then serve in the role of referral liaison and autopsy liaison).

In December 1994, Workshop brochures were mailed to each of the seven Chapter Directors who agreed to recruit at least two staff members or volunteers interested in becoming autopsy educators. Brochures were also sent to each of the liaisons participating in the Network.

\section{Description of the Workshop}

A train-the-trainer model, used extensively in community-based educational efforts, was selected for the workshop. In this model, health professionals or volunteers disseminate information and skills to trainers. The trainers then disseminate the information or skills they learn to interested audiences. Trainers are referred to as peer educators, lay leaders, or community educators. Examples of established programs that are based on the train-the-trainer model include the Arthritis Self-Management program ${ }^{11}$ and "TakePride," a heart disease self-management program for older adults. ${ }^{12}$ Examples of train-the-trainer programs that address issues related to $\mathrm{AD}$ include outreach efforts in the state of Nebraska ${ }^{13}$ and in minority communities in Boston. ${ }^{14}$

The major strength of the train-the-trainer model is the potential for broad dissemination of information and skills after the initial training is completed. Because trainers are usually volunteers from the community, they may be viewed as more acceptable than outside experts and may be better able to tailor a program to the unique needs and interests of a selected target audience. Additionally, trained community residents are more likely to be available on an ongoing basis to answer questions and provide follow-up than outside experts. This may be especially important for sensitive and complex topics like autopsy. 
By providing a comprehensive training program with an opportunity for trainers to observe the program they will be presenting, learn from experts in the field, and gain feedback on their skills, trainers can become a valuable resource in their community. Finally, educational programs can be offered in a variety of sites where people naturally congregate, including shopping malls, churches, senior centers, and other community agencies.

There are also several limitations to the train-thetrainer approach. Few comprehensive evaluations of train-the-trainer programs have been published, limiting an understanding of the factors that are related to program success. Because train-the-trainer programs represent efforts to disseminate information to a broad audience in diverse settings, it is difficult to retain control over all aspects of the information dissemination process, especially when trainers are volunteers. Trainers may modify the content and format of the original program, making it difficult to evaluate the results of their efforts to compare results with those from other similar programs. Trainers may modify evaluation forms or evaluation procedures or choose not to evaluate the programs they offer. Finally, there may be a significant lapse of time between the training and the actual implementation in the community if local agencies (rather than the trainers) are responsible for marketing and scheduling the presentations. Despite these limitations, the train-the-trainer model was chosen because its major strength-the potential for broad dissemination of information and skills-is central to the goals of the AEW.

After attending the Workshop, the newly trained autopsy educators and Network liaisons were expected to become more knowledgeable about autopsy, more aware of the autopsy services in their community and procedures for accessing the Network. Additionally, the autopsy educators were expected to offer presentations about autopsy to interested community groups (i.e., support groups sponsored by local chapters of the Alzheimer's Association) and assist families in the process of decision making about autopsy.

The following topics were addressed during the morning session of the AEW:

- The benefits and barriers to autopsy;

- The removal and examination of the brain tissue;

- Understanding the autopsy report; and

- Planning for an autopsy.

A role play exercise depicting a family in the process of deciding whether or not to have an autopsy performed was also included as part of the Workshop. In addition to the Director of the National Alzheimer's Association's Autopsy Assistance Network, presentations were made by a neuropathologist, neurobiologist, funeral director, and health educators. Separate afternoon sessions were planned for the two target audiences. Staff and volunteers of the local chapters of the Alzheimer's Association attended a session on presentation skills. Network liaisons attended a session designed to help them assist families in planning for an autopsy by solving problem cases illustrating various family situations.

To increase the effectiveness of the Workshop training, a manual was developed for use by the autopsy educators and Network liaisons for their activities in the community. The autopsy manual included the following sections:

- The importance of autopsy to AD research;

- Autopsy for diagnostic and research purposes;

- The autopsy report;

- Family decision-making;

- Planning an autopsy;

- Presentation skills;

- Program evaluation; and

- Reference materials.

In-depth information was developed to supplement several sections of the manual, including a fact sheet on the genetic transmission of $\mathrm{AD}$. Additionally, booklets were developed for Network liaisons that provided step-by-step instructions for assisting families in planning an autopsy.

Selected portions of the AEW were videotaped. These videotapes were edited by staff of the Education Core for use by autopsy educators and Network liaisons. The manual (in combination with the training video) may be useful to others interested in replicating the AEW in their states or communities.

\section{Workshop participants}

Forty people attended the AEW on March 15, 1994, in Lansing, Michigan; 24 representing the local chapters of the Alzheimer's Association and 16 representing the Network. Upon completion of the training, all participants representing the Alzheimer's Association agreed to serve as autopsy educators and all participants representing the Network agreed to serve as liaisons. The purpose of establishing these criteria for participation was to maximize the potential that autopsy information would be disseminated widely.

Workshop participants included eight Chapter Directors of the Alzheimer's Association, 13 Chapter volunteers and staff members, seven referral liaisons, four autopsy liaisons, and eight representatives from various 
state agencies and service providers (e.g., Huntington's Association, geriatric assessment team, nursing home, adult foster care home). The mean age of participants was 45. Eighty-five percent were female and 96 percent were white. The vast majority completed a college or professional degree ( 77 percent).

\section{Evaluation design}

There are several levels of evaluation that are appropriate for a train-the-trainer program. First, a process evaluation of the initial training session can be conducted. For example, participants can provide ratings of their satisfaction with the overall program as well as of the content, format, and presentations. Additionally, pre- and post-tests that assess knowledge, attitudes, and behavioral intentions can be administered before and after the program. The presentation skills of the trainers can also be evaluated, either by an independent observer or an assessment of the trainer's confidence in offering presentations based on the material covered in the program.

A second level of evaluation is conducted when the trainers offer presentations in their communities. As with the initial training session, a process evaluation, pre- and post-tests, and an assessment of presentation skills can be conducted. Finally, the long-term impact of a train-thetrainer program can be evaluated. For example, results of an evaluation of the Arthritis Self-Management program indicate that community residents who attended the training report less pain, less disability, and fewer doctor visits than a comparison sample of adults with arthritis. ${ }^{15}$

For the AEW, evaluation activities at each of these three levels have been conducted or are underway. To evaluate the initial training session, an autopsy knowledge test comprised of seven multiple choice and three true/false items was developed. Because a comprehensive literature search identified no published autopsy knowledge tests with known reliability and validity, the knowledge test used for the present study was designed by health educators in collaboration with the Director of the Education Core to reflect each component of the AEW curriculum.

The multiple choice items assessed the importance, benefits, and cost of autopsy, the contents of the autopsy report, the appropriate people to contact for assistance with autopsy, the type of tissue removed during an autopsy, and the role of the Network in assisting families with autopsy. The true/false items addressed the timing required to plan an autopsy, signing a consent form, and whether autopsies coordinated by the Network are conducted for research and/or diagnostic purposes. The knowledge test was administered in a pre-test/post-test format.

In addition to the autopsy knowledge items, a fivepoint rating scale (poor/excellent) was used for each session of the Workshop: keynote speaker; autopsy educator's program; "Autopsy-Just the Facts;" autopsy report; postmortem network; autopsy planning, coordination and funding; network liaison training; and presentation skills.

Participants were also asked to rate their knowledge of autopsy, level of confidence in discussing autopsy with family members, and level of confidence in their role as a Network liaison before and after the AEW. Finally, participants were asked to provide overall ratings of the workshop and the manual developed for the workshop.

The second level of evaluation of a train-the-trainer program involves the trainers' efforts to disseminate information once they return to their communities. After the AEW, all autopsy educators received a letter encouraging them to present the program at least twice in the next six months. During the workshop, trainers received two evaluation instruments to be completed each time they offered the program. The first instrument, to be completed by the community residents who attended the program, was identical to the pre-/post-test used for the initial training session. The second instrument, to be completed by the trainer who offered the program, included a section to provide a description of when and where the program took place, ratings of how well the training and manual prepared them to make the presentation, and a rating of their ability to discuss autopsy with caregivers and other family members (using a five-point scale, poor/excellent). Three open-ended questions were also included:

- What questions/issues were raised by program participants?

- Overall, how did you feel about the program? and

- How would you change your presentation based on your experience?

All evaluation instruments were to be mailed to the staff of the Education Core. To assess the process of information dissemination as a result of the workshop, a tracking form was developed to record the following information about each presentation conducted, including the date, type of group, number of participants, and the number of participants and presenter evaluations received.

\section{Evaluation results}

\section{Initial training session}

Based on 19 participants for whom a matched pre-test and post-test were completed, results indicate that there 
was significant knowledge change for six of the 10 autopsy knowledge items. Specifically, a greater percentage of correct responses was obtained at post-test compared to pre-test (all p's $\leq .05$ ) for items that assess:

- The time required to plan an autopsy (58 percent correct at pre-test compared to 83 percent at post-test);

- The process of gaining consent (58 percent vs. 94 percent);

- Autopsy procedure (63 percent vs. 89 percent);

- The cost of autopsy services ( 22 percent vs. 63 percent);

- Staffing of the Network (47 percent vs. 72 percent); and

- The purpose of autopsies coordinated by the Network ( 58 percent vs. 89 percent).

For each of the remaining four knowledge items (that assess the role of autopsy in $\mathrm{AD}$, the benefits of autopsy, the contents of an autopsy report, and the most appropriate person to assist families in planning an autopsy), the percentage of correct responses at post-test was higher than at pre-test, but the change was not significant. In each case, the percentage of participants with correct responses at pre-test was high (at least 75 percent), limiting the magnitude of possible change from pre-to post-test.

Average ratings for each session of the AEW range from 3.9 (corresponding with very good) for the session on presentation skills to 4.8 (corresponding to excellent) for the informational session on autopsy and autopsy procedures. At least 80 percent of participants rated each section as "very good' or "excellent."

One fourth of participants ( 26 percent) rated their knowledge of autopsy as very good and 7 percent as excellent before the AEW, compared to 56 percent as very good and 30 percent as excellent after the AEW (see Figure 1). Less than one third ( 30 percent) of participants rated their confidence in discussing autopsy with family members as very good, while no one rated their confidence as excellent before the AEW. After the AEW, three fourths (74 percent) rated their confidence as very good and 7 percent rated their confidence in discussing autopsy with family members as excellent (see Figure 2).

Two thirds of the participants (67 percent) rated the workshop as excellent, 26 percent as very good and 7 percent as good. The autopsy manual was rated by 89 percent of participants as excellent and as very good by 11 percent.

Presentations by trainers in the community

In March 1994, 24 autopsy educators representing 11 local chapters of the Alzheimer's Association received the AEW training. Approximately six months later, seven of the 24 trainers completed a total of 12 autopsy education presentations, reaching approximately 120 people. Evaluation instruments were returned for only three of the 12 programs. Because the number of presentations made and evaluation instruments returned by the trainers was much lower than expected, we conducted a telephone survey with 15 randomly selected trainers to determine barriers to presenting the autopsy education program. The most frequently mentioned barriers included:

- The trainer's busy schedule, limiting the time available to present the program;

- Difficulty in finding interested groups;

- The sensitive and emotional nature of autopsy; and

- The competing demands of the chapters, with autopsy being a relatively low priority.

All of the trainers who responded to the survey reported that they plan to offer autopsy education programs in the future; most reported that the autopsy materials distributed at the workshop had been useful when talking with individual family members about autopsy. Additionally, respondents reported that the information provided in the autopsy manual was used by chapter staff to write newsletter articles, inform advisory boards and train helpline staff.

\section{Long-term impact of the AEW}

The trainers progress in making autopsy education presentations will be followed for the year following the AEW. In addition to gathering information about the presentations conducted in the community, each of the local chapters has been asked to keep track of the number of autopsies their staff assists with. The Network coordinator will also track the number of autopsies facilitated by the autopsy liaisons and referral liaisons who attended the AEW.

\section{Discussion}

Despite the important role of autopsy in confirming diagnoses and advancing research in Alzheimer's disease, limited attention has been afforded to autopsy education. The present study was conducted to describe the design, implementation, and evaluation of a statewide autopsy education intervention based on a train-the-trainer model conducted in collaboration with the local chapters of the Alzheimer's Association in Michigan and the Michigan Postmortem Dementia Network.

As a result of the AEW, 40 volunteers have been trained to disseminate accurate information about 
Figure 1. Participant's ratings of their knowledge of antopsy before the workshop (at pre-test) and after the workshop (at post-test).

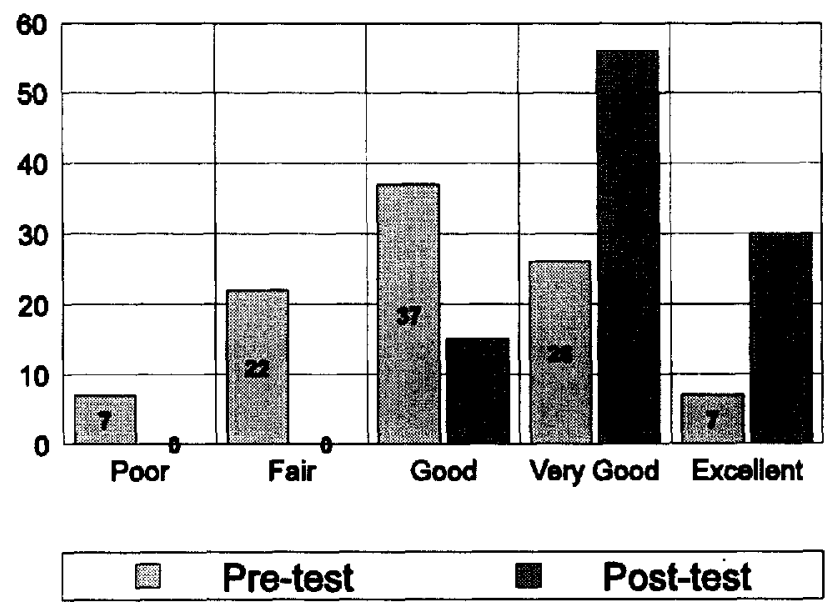

autopsy, respond to questions and concerns of family members, and assist families in accessing the Michigan Dementia Postmortem Network. The AEW participants represent all areas of the state, including many rural and underserved areas. The AEW was well-received, with most sessions being rated as very good or excellent. The vast majority of participants rated the manual as excellent. Participants reported significant improvements in knowledge about autopsy and confidence in discussing autopsy after the AEW.

The greatest challenge for those implementing trainthe-trainer programs occurs once the trainers return to their communities. In the case of the AEW, the process of information dissemination after the initial training session was much slower than expected. In part, this process was delayed because of the nature of the training-autopsy education. Autopsy is typically not a topic in high demand by community groups because it is a sensitive and personal issue and is usually a concern only for a small segment of the general population (e.g., family members of people with a dementing illness). Additionally, because most trainers offer programs to existing groups, they need to conform to pre-established meeting schedules. For example, most Alzheimer's Association support groups do not meet during the summer and most groups have their meeting agenda set several months in advance. Finally, most of the local chapters of the Alzheimer's Association in Michigan face serious budget constraints and personnel shortages. Although autopsy is an important issue, it may be a relatively low priority compared to providing general information and referral services. In combination, these factors may limit the number of pro- grams offered, restrict the marketing and publicity for individual programs, and result in delays between the initial training and implementation in the community.

In light of the barriers to implementing autopsy education programs, several recommendations can be made to maximize the success of future interventions. First, the process of information dissemination should be followed for at least one year after the initial training session. If follow-up is limited to a shorter period, a high proportion of the dissemination activities may be missed. Second, the collaborative relationship between the trainers and the staff responsible for planning the program should be maintained long after the program is implemented. In the case of the AEW, the trainers remain in close contact with staff of the Education Core and have agreed to evaluate the autopsy presentations they make in their community. They will also provide information about the number of families that request information about autopsy and the number of autopsies their chapter assists with. This information will be very valuable in assessing the long-term impact of the AEW. In addition to offering periodic presentations about autopsy in their local communities, the trainers representing the local chapters should be encouraged to include autopsy education as part of their general caregiver training program. The evaluation for the present AEW was limited to an assessment of knowledge gains and trainee's self-reported confidence in making presentations about autopsy. Due to the limited resources available for evaluation and the time constraints of a one-day workshop, the trainer's presentation skills were not evaluated as part of this study. An evaluation of presentation skills is highly recommended as part of the train-the-trainer model. 


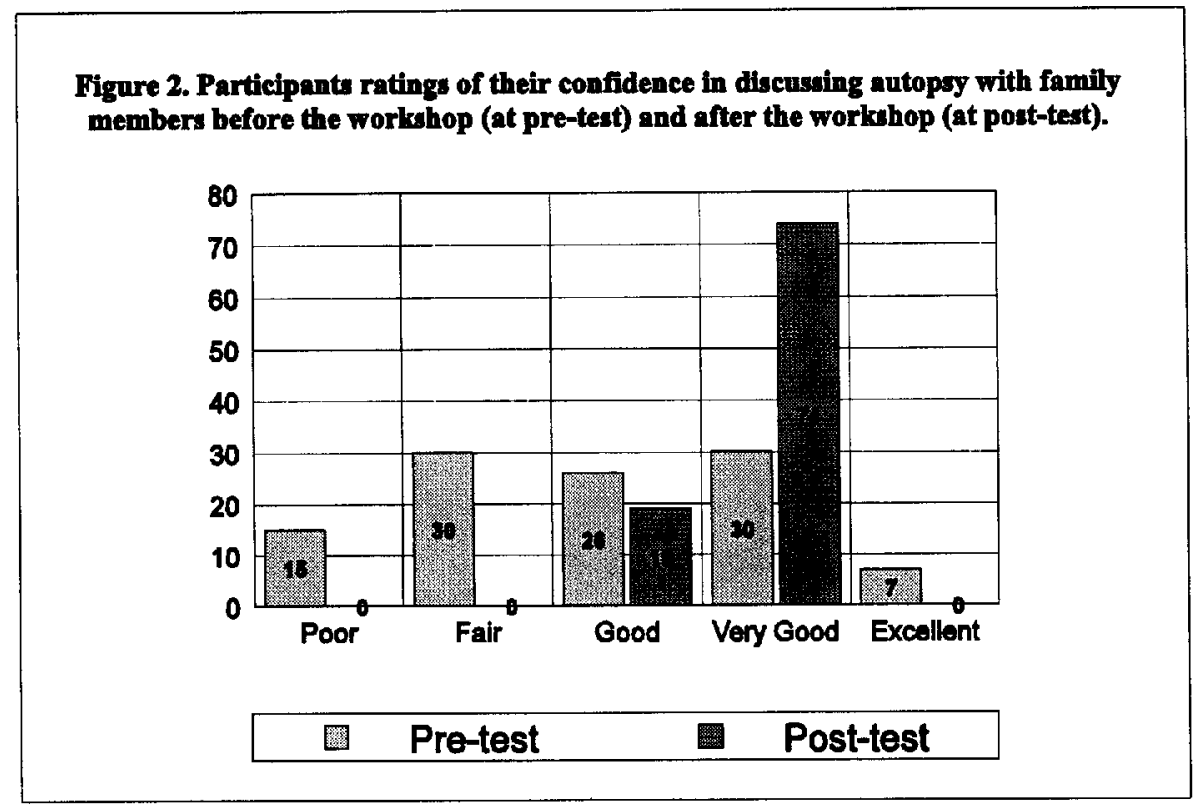

The autopsy manual was developed as a resource for the trainers. It has been very useful to chapter staff and Network volunteers for writing newsletter articles, informing advisory boards about autopsy, and training helpline staff. Although recording these activities was not part of the original evaluation design, these broad avenues of dissemination represent a very important outcome of the Workshop.

\section{References}

1. Nemetz. P. N., Beard, C. M., Ballard, D. J, Ludwig, J., Tangalos, E. G., Kokmen, E., Weigel, K. M., Belau, P. G. Boume, W. M., \& Kurland, L. T. (1989). Resurrecting the autopsy: Benefits and recommendations. Mayo Clinic Proceedings. 64, 1065-1076.

2. Souder, E., \& Trojanowski, J. Q. (1992). Autopsy: Cutting away the myths. Journal of Neuroscience Nursing, 24, 134- 139.

3. Price, R. A., \& McCormick, W. F. (1981). The declining autopsy rate and its significance for neuropathology: Two viewpoints. Journal of Neuropathology and Experimental Neurology, 40, 489-492.

4. Powers, R. E.. Powell, S. K., Schlough. C. N., \& Whitehouse, P.J. (1989) Autopsy services for dementia patients. The Gerontologist, 99, 120-123. 5. Connell, C. M, Avey, H., \& Holmes. S. B. (1994). Attitudes about autopsy: Implications for educational interventions. The Gerontologist, 34, 665-673.

6. McPhee, S. J., Bottles, K., Lo, B., Saika. G., \& Crommie, D. (1986). To redeem them from death: Reactions of family members to autopsy The American Journal of Medicine. 80-7 665-671.

7. Whitehouse, P.J. (1993). Autopsy. The Gerontologist 33, 436.

8. Post, S. G. (1994). Genetics, ethics, and Alzheimer disease. Journal of the American Geriatrics Society, 42, 782-786.

9. Witter, D. M., Tolle, D. M., \& Mosley, J. R. (1990). A bereavement program: Good care, quality assurance, and risk management. Hospital \& Health Services Administration, 35, 263-274.

10. Shope, J. T., Holmes, S. B., Hogan, J., Tang, G., Izenson, S., Gilman, S., \& Jones, M.Z. (1993). Pathologists participation in postmortem examinations for patients with dementia. The Gerontologist, 33, 461-467.

11. Lorig, K., Laurin, J., \& Holman, H. R. (1984). Arthritis self-management: A study of the effectiveness of patient education for the elderly. The Gerontologist. 24, 455-457.

12. Clark, N.M., Janz, N. K., Becker, M. H., Schork, M. A., Wheeler,
J., Liang, J., Dodge, J.A., Keteyian, S., Rhoads, K.L., \& Santinga, J.T. (1992). Impact of self-management education on the functional health status of older adults with heart disease. The Gerontologist. 32, 438-443. 13. Potter, J.F.\& Foster, B.G. Reaching nural communities with Alzheimer s education. The Gerontologist, 34, 386.

14. Belleville-Taylor, P., Lombardo, N., Levkoff, S., Gornstein, E., Rosenberg, R., \& Ooi, W.L. (1994). Outreach education to inner city Boston healthcare workers and black caregivers of elders with dementia. The Gerontologist, 34, 386.

15. Lorig, K. R., Mazonson, P. D., \& Holman, H. R. (1993). Evidence suggesting that health education for self-management in patients with chronic arthritis has sustained health benefits while reducing health care costs. Arthritis and Rheumatism, 36, 439-446.

\section{Acknowledgments}

The authors would like to thank Doris Barry, Mary Frenza, Shirley Heenan, Yvonne Jonkers, Erma McMillan, Margie Trumble, and Chris Weaner, members of the planning committee; the local chapters of the Alzheimer's Association in Michigan; June White of the National Alzheimer's Association; Margaret Z. Jones and Connie D'Amato of the Michigan Dementia Postmortem Network; and Pegeen McGhan for her assistance with manuscript preparation. This research was supported in part by a grant by the US Public Health Service (NIH NIA \#P50A608671) to the Education Core of the Michigan Alzheimer's Disease Research Center, Cathleen M Connell, Principal Investigator.

\section{Note}

Address correspondence to Cathleen $\mathrm{M}$ Connell, $\mathrm{PhD}$, Director, Education and Information Transfer Core, Michigan Alzheimer's Disease Research Center, 300 North Ingalls Building, University of Michigan, Ann Arbor, MI 48109-0489. 